\title{
Influence of ghrelin gene polymorphisms on hypertension and atherosclerotic disease
}

\author{
Heiner K Berthold ${ }^{1,2}$, Eleni Giannakidou ${ }^{1,3}$, Wilhelm Krone ${ }^{3}$, David-Alexandre Trégouët ${ }^{4}$ \\ and Ioanna Gouni-Berthold ${ }^{3}$
}

Ghrelin is involved in several metabolic and cardiovascular processes. Recent evidence suggests its involvement in blood pressure regulation and hypertension. The aim of the study was to determine associations of single-nucleotide polymorphisms (SNPs) and haplotypes of the ghrelin gene (GHRL) with hypertension and atherosclerotic disease. Six GHRL SNPs (rs27647, rs26802, rs34911341, rs696217, rs4684677 and a -473G/A (with no assigned rs/D)) were investigated in a sample of 1143 hypertensive subjects and 1489 controls of Caucasian origin. Both single-locus and haplotype association analyses were performed. In single-locus analyses, only the non-synonymous rs34911341 was associated with hypertension (odds ratio $(\mathrm{OR})=1.95$ (95\% confidence interval $(\mathrm{Cl}): 1.26-3.02), P=0.003$ ). Six common haplotypes with frequency $>1 \%$ were inferred from the studied GHRL SNPs, and their frequency distribution was significantly different between hypertensive subjects and controls $\left(\chi^{2}=12.96\right.$ with 5 d.f. (degree of freedom), $\left.P=0.024\right)$. The effect of rs26802 was found to be significantly $(P=0.017)$ modulated by other GHRL SNPs, as its C allele conferred either an increased risk (OR=1.30 (1.08-1.57), $P=0.005)$ or a decreased risk ( $O R=0.50(0.23-1.06), P=0.07)$ of hypertension according to the two different haplotypes on which it can be found. No association of GHRL SNPs or haplotypes with atherosclerotic disease was observed. In conclusion, we observed statistical evidence for association between GHRL SNPs and risk of hypertension.

Hypertension Research (2010) 33, 155-160; doi:10.1038/hr.2009.194; published online 11 December 2009

Keywords: ghrelin; haplotype analysis; polymorphism

\section{INTRODUCTION}

Ghrelin, a 28-amino acid peptide from the stomach, is an endogenous ligand for the growth hormone secretagogue receptor (GHS-R1a) that stimulates growth hormone release. ${ }^{1}$ Recent findings have expanded the actions of ghrelin to include metabolic, immunologic, orexigenic, reproductive and behavioral effects, as well as modulation of gastrointestinal motility. ${ }^{2,3}$ Moreover, there is increasing evidence for a cardiovascular function of ghrelin. Ghrelin has been postulated to exert a protective effect against atherosclerosis by anti-inflammatory, ${ }^{4}$ vasodilatory ${ }^{5}$ and antioxidant effects, ${ }^{4}$ as well as through improved endothelial function. ${ }^{6}$ Furthermore, a radiolabeled ghrelin [ $\left.{ }^{125} \mathrm{I}-\mathrm{His} 9\right]$ has been shown to bind to the heart and peripheral vascular tissues. The signal was augmented in atherosclerotic regions, suggesting that ghrelin receptor expression is upregulated in such areas, thus implicating ghrelin in the development of atherosclerosis. ${ }^{7}$ Expression of both ghrelin and its receptor has been identified in the heart and blood vessels, and a decrease in blood pressure was observed after intravenous injection of ghrelin in humans. ${ }^{1}$ Moreover, low plasma ghrelin levels have been found to be associated with hypertension in a Finnish cohort of hypertensive subjects. ${ }^{8}$ In contrast, high ghrelin concentrations were associated with increased mean intima-media thickness in Finnish males. ${ }^{9}$

Recently, several single-nucleotide polymorphisms (SNPs) of the ghrelin gene $(G H R L)$ have been shown to be associated with blood pressure levels. ${ }^{10}$ In particular, the combination of the most common genotypes at rs27647, rs26802, rs696217 (Leu72Met) and rs4684677 (Gln90Leu) was associated with the lowest systolic and diastolic blood pressure levels in a sample of Finnish subjects with impaired glucose tolerance. ${ }^{10}$ In addition, in another Finnish study, the Gln51 allele of the non-synonymous rs34911341 (Arg51Gln) SNP was associated with an increased risk of hypertension. ${ }^{11}$ The proposed underlying mechanism involved its association with either low IGF-I concentrations (as IGF-I has been reported to stimulate endothelial nitric oxide formation) or lower circulating ghrelin levels, as previously reported for this SNP. ${ }^{11}$

However, little is known about the potential association of these GHRL SNPs with atherosclerosis and its cardiovascular complications. To our knowledge, only rs696217 has been examined in relation to coronary heart disease (CHD) without positive findings, ${ }^{12,13}$ but no data are available about the association of this variant or any other

${ }^{1}$ Lipid Clinic, Interdisciplinary Metabolism Center, Charité University Medicine Berlin, Virchow Clinic Campus, Berlin, Germany; ${ }^{2}$ Department of Geriatrics, Charité University Medicine Berlin, Berlin, Germany; ${ }^{3}$ Department of Internal Medicine II, University of Cologne, Cologne, Germany and ${ }^{4}$ INSERM UMRS_937, UPMC Univ Paris 06, UMR_S 937, Paris, France

Correspondence: Professor HK Berthold, Lipid Clinic, Interdisciplinary Metabolism Center, Charité University Medicine Berlin, Virchow Clinic Campus, Augustenburger Platz 1, Berlin 13353, Germany.

E-mail: heiner.berthold@charite.de

Received 18 August 2009; revised 26 September 2009; accepted 27 October 2009; published online 11 December 2009 
GHRL SNPs with overall atherosclerotic disease (including other manifestations beyond CHD).

The aim of this study, then, was to explore the influence of previously studied GHRL SNPs on hypertension and atherosclerotic disease in a large cohort of Caucasian subjects by means of singlelocus analyses, as done in previous studies, while also using more efficient haplotype analyses.

\section{METHODS}

\section{Study population}

A total of 2632 Caucasian subjects (54.5\% male, age $57 \pm 12$ years, range: 15-100) were included in the study. Their data were obtained from the Lipid Analytic Cologne (LIANCO), a database described elsewhere. ${ }^{14,15}$ In brief, LIANCO was designed to assess the relationships between genetic mutations, serum lipoproteins, other biochemical parameters and clinical data on hypertension, diabetes and atherosclerotic disease. Approval of the study protocol was obtained from the Ethics Committee of the University of Cologne. Between spring 1999 and March 2002, a total of about 5000 patients were recruited in the Cologne (Germany) area by hospitals and office-based physicians.

Subjects with hypertension $(N=1143,52.5 \%$ male, age $60 \pm 12$ years $)$ and normotensive controls ( $N=1489,56.0 \%$ male, age $55 \pm 13$ years) participated in this case-control study. Hypertension was operationally defined as having a measured systolic blood pressure of $>140 \mathrm{~mm} \mathrm{Hg}$ and/or diastolic blood pressure of $>90 \mathrm{~mm} \mathrm{Hg}$; patients taking antihypertensive drugs were also classified as hypertensive, irrespective of their measured blood pressure. Subjects were on different antihypertensive medications, including diuretics (11.1\%), angiotensin-converting enzyme inhibitors (17.0\%), angiotensin II receptor 1 antagonists (5.8\%), calcium channel blockers (7.1\%), $\beta$-blockers (18.8\%), $\alpha$-blockers (1.3\%), antihypertensive agents with central mechanisms of action $(0.5 \%)$, peripheral vasodilators $(0.2 \%)$ and combination therapies. Altogether, about $39.6 \%$ of the participants in the hypertension group were on antihypertensive drug treatment.

Atherosclerotic disease was defined as the presence and/or history of at least one of the following parameters: CHD, stroke, transient ischemic attack, prolonged reversible ischemic neurological deficit or peripheral arterial vascular disease. CHD was defined as the presence of at least one of the following conditions: angiographic evidence of $\mathrm{CHD}$, myocardial infarction, angina pectoris, coronary bypass surgery or positive stress test. Subjects were defined as diabetic through oral glucose tolerance test $(2 \mathrm{~h}$ plasma glucose concentration $\geqslant 200 \mathrm{mg} \mathrm{dl}^{-1}\left(\geqslant 11.1 \mathrm{mmoll}^{-1}\right)$ ) or if receiving antidiabetic drug treatment. The baseline characteristics of the study population are given in Table 1.

\section{Genotyping}

Six GHRL SNPs were investigated in this report: rs27647, rs26802, rs696217, rs4684677, rs34911341 and a G/A SNP located at position -473 from the transcription site (referred to as $-473 \mathrm{G} / \mathrm{A}$, as it has not yet been assigned an rsID). Genomic DNA was prepared from peripheral blood using standard techniques. The six SNPs were detected by PCR followed by restriction fragment length polymorphism as previously described. ${ }^{10}$

The genomic DNA was amplified by PCR followed by digestion with specific restriction enzymes (Fermentas, St Leon-Rot, Germany), as previously described. ${ }^{10}$ The PCR products were automatically sequenced (ABI Prism Genetic Analyzer model 310, Applied Biosystems, Foster City, CA, USA). The sequence of both strands was determined.

Quality checks to ensure correctness of the genotypes were carried out by independent rating of the results by two investigators. Discrepancies were resolved by either reaching consensus or re-genotyping. Furthermore, to assess genotyping reproducibility, a random $10 \%$ of the samples were re-genotyped, yielding $100 \%$ concordance. The percentage of missing genotype data ranged from 0 to $0.7 \%$ (mean value $0.3 \%$ ) for the six SNPs.

\section{Statistical analysis}

Descriptive statistics are given, unless otherwise indicated, as proportions (percent or mean values \pm s.d.). As a large proportion of the patients were also being treated with lipid-lowering drugs, we corrected the low-density lipoprotein cholesterol levels according to previously described methods ${ }^{14}$ to obtain values simulating untreated conditions. Triglyceride concentrations were $\log$-transformed before analysis. Contingency tables and $\chi^{2}$-tests were used to analyze differences between proportions. Comparison of means was performed by the unpaired Student's $t$-test or by the Mann-Whitney $U$-test. Allele frequencies were estimated by gene counting, and SNP case-control comparisons were tested using the Cochran-Armitage trend test.

Statistical analyses were carried out using the Statistical Package for the Social Sciences Version 16.1.2 (SPSS, Munich, Germany), except for linkage

Table 1 Subjects' characteristics

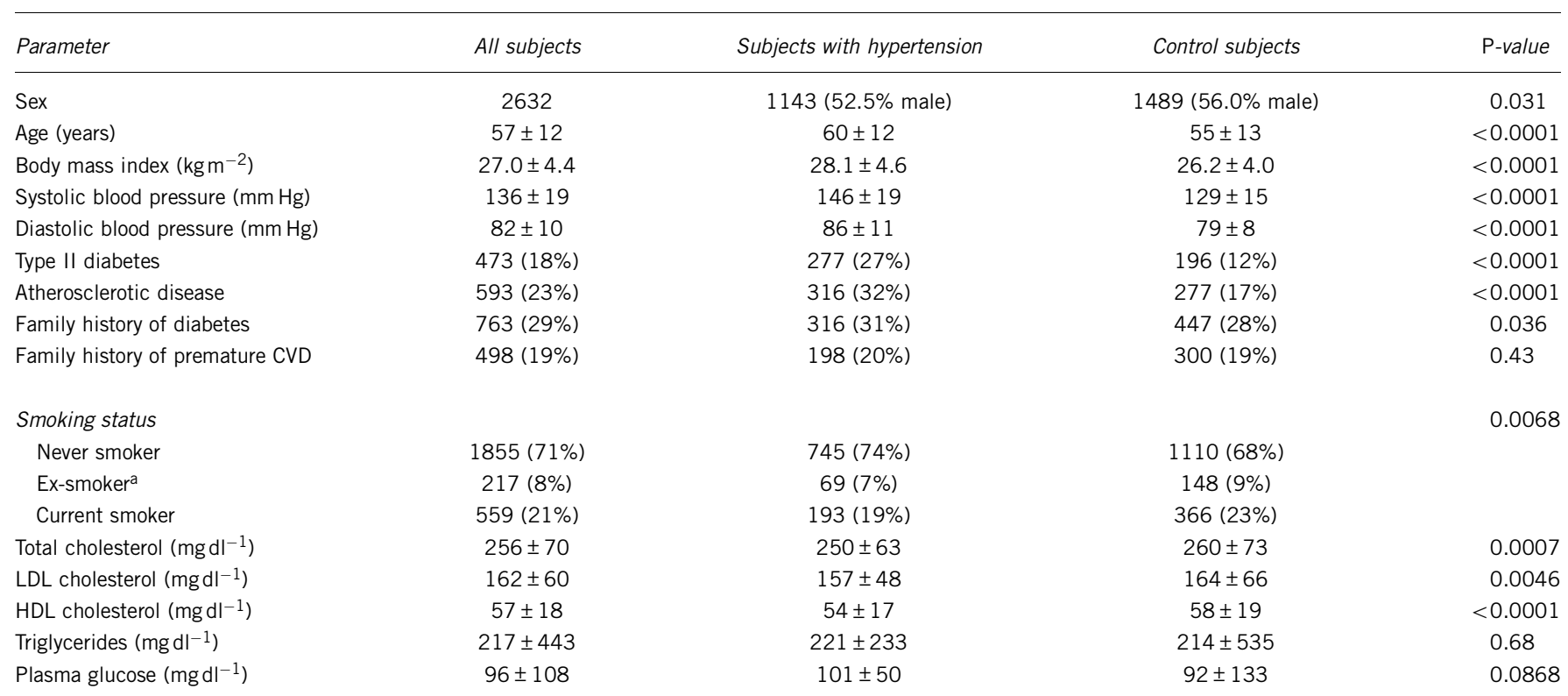

Abbreviations: CVD, cardiovascular disease; HDL, high-density lipoprotein; LDL, low-density lipoprotein. a Having stopped smoking for $>1$ year. 
disequilibrium and haplotype analyses, which were carried out using the THESIAS software. ${ }^{16}$ All haplotype analyses were adjusted for age, gender, body mass index, diabetes and atherosclerotic disease when appropriate. Because the natural history of hypertension and atherosclerotic disease differs in males and females, ${ }^{17}$ analyses were carried out separately in males and females, and the Mantel-Haenszel statistic was used for testing the homogeneity of the results across gender.

To deal with the number of tested SNPs, statistical significance was defined as $P<0.01$ ( $=0.05 / 5,5$ being the number of SNPs apart from rs4684677, which was in complete association with rs696217). All tests were two-sided.

\section{RESULTS}

For ease of presentation, the results presented are those obtained in the pooled sample of males and females after checking for the homogeneity of the associations across gender.

\section{Association of GHRL SNPs with hypertension}

Genotype and allele frequencies of the six GHRL SNPs are presented in Table 2. In the whole cohort, all observed genotype distributions were compatible with Hardy-Weinberg equilibrium, and only rs26802 showed a slight deviation from Hardy-Weinberg equilibrium in hypertensive patients $(P=0.036)$. Only rs34911341 showed an association with hypertension, in which carrying the Gln51 allele was associated with an increased risk of hypertension (odds ratio $(\mathrm{OR})=1.761$ (95\% confidence interval (CI): 1.151-2.695), $P=0.0084$ ). After adjusting for age, gender, smoking, body mass index and diabetes, this OR was still significant (OR 1.949 (1.257-3.021), $P=0.003$ ).

As indicated in Table 3, little pair-wise linkage disequilibrium was observed between GHRL polymorphisms, except between the rs696217 and rs4684677 variants that were in complete association. As a consequence, results involving rs4684677 are not shown in this report, as they are exactly the same as those observed for rs696217. Six haplotypes with frequencies $>1 \%$ were inferred from the three common GHRL SNPs (rs27647, rs26802 and rs696217) and accounted for more than $99 \%$ of the whole chromosome (Table 4). The frequency distribution of these six haplotypes significantly differed between cases and controls $\left(\chi^{2}=12.96\right.$ with 5 d.f. (degree of freedom), $P=0.024)$. Although the AALeu haplotype was less frequent in cases than in controls $(0.261$ vs. 0.293$)$, the opposite was observed for the ACLeu haplotype $(0.268 v$ s. 0.244$)$. These two haplotypes only differ at the rs26802 position, suggesting that the rs $26802 \mathrm{C}$ allele confers an increased risk of hypertension $(\mathrm{OR}=1.303(1.083-1.567), P=0.005)$ when carried on the A-Leu background. Conversely, on the A-Met background, the rs $26802 \mathrm{C}$ allele tended to be associated with a decreased risk of hypertension (OR $0.496(0.231-1.061), P=0.071)$, as the ACMet haplotype was nearly half as frequent in cases as in controls (0.012 vs. 0.021), and the frequency of the AAMet haplotype was quite similar in cases and in controls $(0.054$ vs. 0.050). The test for homogeneity of these two ORs was significant $\left(\chi^{2}=5.66\right.$ with 1 d.f.,

Table 3 Pairwise LD between GHRL polymorphisms in the pooled sample $(n=2633)$

\begin{tabular}{lcccccc}
\hline & $r s 27647$ & $r s 26802$ & $-473 G / A$ & $r s 34911341$ & $r s 696217$ & $r s 4684677$ \\
\hline rs27647 & - & -0.638 & 0.185 & -0.371 & -0.835 & -0.835 \\
rs26802 & 0.124 & - & 0.048 & -0.453 & -0.087 & -0.087 \\
$-473 G / A$ & 0.001 & 0.000 & - & -1 & -1 & 1 \\
rs34911341 & 0.002 & 0.002 & 0.000 & - & -1 & -1 \\
rs696217 & 0.036 & 0.000 & 0.000 & 0.001 & - & 1 \\
rs4684677 & 0.036 & 0.000 & 0.000 & 0.001 & 1 & - \\
\hline
\end{tabular}

Abbreviation: LD, linkage disequilibrium.

In the upper-right triangle of the table, LD is expressed in terms of $D^{\prime}$, which is the ratio of the unstandardized coefficient to its maximal/minimal value, whereas in the lower-left triangle of this matrix, LD is expressed in terms of the $r^{2}$ statistic.

Table 2 Genotype and allele frequencies of ghrelin polymorphisms in hypertensive patients and non-hypertensive controls

\begin{tabular}{|c|c|c|c|c|c|c|}
\hline SNP & Group & & e frequencies, & & $M A F$ & $P$-value ${ }^{a}$ \\
\hline \multirow[t]{3}{*}{ rs27647 } & & GG & $\mathrm{GA}$ & $\mathrm{AA}$ & $A$ & \\
\hline & Hypertensives & $178(17.2)$ & $482(46.5)$ & $376(36.3)$ & 0.596 & 0.364 \\
\hline & Controls & $225(15.4)$ & $695(47.5)$ & $542(37.1)$ & 0.608 & \\
\hline \multirow[t]{3}{*}{ rs26802 } & & $A A$ & $A C$ & $\mathrm{CC}$ & C & \\
\hline & Hypertensives & $454(43.6)$ & $490(47.0)$ & $98(9.4)$ & 0.329 & 0.090 \\
\hline & Controls & $696(47.4)$ & $641(43.7)$ & $130(8.9)$ & 0.307 & \\
\hline \multirow[t]{3}{*}{$-473 G / A$} & & $\mathrm{GG}$ & $\mathrm{GA}$ & AA & $A$ & \\
\hline & Hypertensives & $1014(97.1)$ & $30(2.87)$ & 0 & 0.014 & 0.743 \\
\hline & Controls & $1429(97.3)$ & $39(2.66)$ & 0 & 0.013 & \\
\hline \multirow[t]{3}{*}{ rs34911341 } & & Arg51Arg & Arg51GIn & Gln51GIn & Gln51 & \\
\hline & Hypertensives & 993 (95.3) & $49(4.70)$ & 0 & 0.024 & 0.0084 \\
\hline & Controls & $1428(97.3)$ & $40(2.73)$ & 0 & 0.014 & \\
\hline \multirow[t]{3}{*}{ rs696217 } & & Leu72Leu & Leu72Met & Met72Met & Met72 & \\
\hline & Hypertensives & $980(86.2)$ & 154 (13.5) & $3(0.26)$ & 0.070 & 0.439 \\
\hline & Controls & $1265(85.4)$ & 207 (14.0) & $9(0.61)$ & 0.076 & \\
\hline \multirow[t]{3}{*}{ rs4684677 } & & Gln90GIn & GIn90Leu & Leu90Leu & Leu90 & \\
\hline & Hypertensives & $980(86.2)$ & $154(13.5)$ & $3(0.26)$ & 0.070 & 0.439 \\
\hline & Controls & $1265(85.4)$ & 207 (14.0) & $9(0.61)$ & 0.076 & \\
\hline
\end{tabular}

Abbreviation: MAF, minor allele frequency.

aCochran-Armitage test $P$-value. 
Table 4 Haplotype frequencies distribution of GHRL polymorphisms in hypertensive and control population

\begin{tabular}{|c|c|c|c|c|c|c|}
\hline \multicolumn{3}{|c|}{ Polymorphisms } & \multicolumn{2}{|c|}{ Haplotype frequencies } & \multirow[b]{2}{*}{ Haplotypic OR $(95 \% C l)^{\text {a }}$} & \multirow[b]{2}{*}{ P-value } \\
\hline rs27647 & rs26802 & rs696217 & Controls $(\mathrm{n}=1489)$ & Hypertensives $(n=1143)$ & & \\
\hline G & C & Leu & 0.039 & 0.048 & $1.189[0.842$ to 1.681$]$ & 0.325 \\
\hline A & A & Leu & 0.293 & 0.261 & $0.883[0.749$ to 1.039$]$ & 0.135 \\
\hline A & A & Met & 0.050 & 0.054 & $1.108[0.825$ to 1.489$]$ & 0.494 \\
\hline
\end{tabular}

Abbreviations: $\mathrm{BMI}$, body mass index; $\mathrm{Cl}$, confidence interval; d.f., degree of freedom; OR, odds ratio.

The rs4684677 is not shown in this table as it was in complete association with the rs696217. The $-473 \mathrm{G} / \mathrm{A}$ and rs34911341 polymorphisms are not shown in this table because their rare alleles were inferred to be present on several very rare $(<1 \%)$ haplotypes.

${ }^{a}$ Haplotypic OR with their $95 \% \mathrm{Cl}$ adjusted for age, sex and BMI. These are haplotypic ORs by comparison with the most frequent GALeu haplotype, under the assumption of haplotypic additive effects. The global test of haplotypic association was significant $\left(\chi^{2}=12.96\right.$ with 5 d.f., $\left.P=0.024\right)$.

Table 5 Association of GHRL polymorphisms with SBP and DBP

\begin{tabular}{|c|c|c|c|c|c|}
\hline & \multirow{2}{*}{$N^{a}$} & \multicolumn{2}{|c|}{$S B P$} & \multicolumn{2}{|c|}{$D B P$} \\
\hline & & Controls & Hypertensives & Controls & Hypertensives \\
\hline$A A$ & $542 / 376$ & $124.6(8.38)$ & $148.1(16.2)$ & $77.6(5.23)$ & $86.6(8.97)$ \\
\hline$A G$ & $696 / 482$ & $125.0(7.29)$ & $146.8(15.3)$ & $78.0(4.74)$ & $86.7(8.68)$ \\
\hline GG & $225 / 178$ & $124.3(8.58)$ & $147.2(15.2)$ & $77.3(6.11)$ & $86.9(10.0)$ \\
\hline \multicolumn{6}{|l|}{ rs26802 } \\
\hline $\mathrm{AA}$ & $696 / 454$ & $124.8(8.24)$ & $146.8(15.8)$ & $77.6(5.28)$ & $86.4(9.03)$ \\
\hline$A C$ & $642 / 490$ & $124.4(7.80)$ & $147.1(15.5)$ & $77.8(5.04)$ & $86.7(8.98)$ \\
\hline $\mathrm{CC}$ & $130 / 98$ & $126.0(6.85)$ & $149.8(15.4)$ & $78.0(5.45)$ & $87.5(9.36)$ \\
\hline$P^{\mathrm{b}}$ & & $P=0.045$ & $P=0.267$ & $P=0.701$ & $P=0.425$ \\
\hline \multicolumn{6}{|l|}{$-473 G / A$} \\
\hline \multicolumn{6}{|c|}{ rs34911341 } \\
\hline ArgArg & $1428 / 993$ & $124.8(7.92)$ & $147.2(15.6)$ & 77.7 (5.18) & $86.6(9.07)$ \\
\hline ArgGln & $40 / 49$ & $123.9(8.77)$ & $147.5(17.1)$ & $78.0(5.67)$ & $87.4(8.38)$ \\
\hline$p$ & & $P=0.470$ & $P=0.783$ & $P=0.795$ & $P=0.473$ \\
\hline \multicolumn{6}{|l|}{ rs696217 } \\
\hline LeuLeu & $1265 / 980$ & $124.7(7.94)$ & $146.9(15.9)$ & 77.7 (5.27) & $86.3(9.20)$ \\
\hline LeuMet & $207 / 154$ & $124.9(7.58)$ & $146.3(14.1)$ & $78.2(4.72)$ & 86.7 (8.39) \\
\hline MetMet & $9 / 3$ & $126.0(12.2)$ & $140.0(17.3)$ & 76.8 (3.98) & $83.3(5.77)$ \\
\hline$P$ & & $P=0.957$ & $P=0.701$ & $P=0.362$ & $P=0.911$ \\
\hline
\end{tabular}

Abbreviations: DBP, diastolic blood pressure; d.f., degree of freedom; SBP, systolic blood pressure.

Mean (s.d.) are shown. Test were performed on adjusted values for age, gender, body mass index, diabetes and atherosclerotic disease.

aNumber of controls/number hypertensives according to genotypes.

bGenotypic test of association (2 d.f.).

cTest of association with 1 d.f., comparing carriers of the rare allele with noncarriers.

$P=0.017$ ), suggesting that the effect of the rs26802 polymorphism on the risk of hypertension could be modulated by other GHRL polymorphisms. After adjusting for these haplotypic effects, the effect of the rs34911341 polymorphism was still significant $(\mathrm{OR}=1.934$ (1.2103.092), $P=0.006)$.
Association of GHRL SNPs with blood pressure, atherosclerosis and cardiovascular risk factors

No single GHRL SNPs (Table 5) or haplotypes (data not shown) were associated with systolic or diastolic blood pressure. Similar negative results were observed when patients taking antihypertensive drugs 
were excluded from the analysis (data not shown). No association was observed with atherosclerotic disease, body mass index, glucose plasma concentrations or lipoprotein concentrations (Supplementary information).

\section{DISCUSSION}

In this report, six GHRL SNPs were studied in relation with the risk of hypertension in a sample of Caucasian subjects.

One non-synonymous SNP, rs34911341 (Arg51Gln), was found to be associated with hypertension, as carriers of the Gln51 allele had an approximately twofold greater risk of hypertension than noncarriers. This result is in complete agreement with that observed by Poykko et al. ${ }^{11}$ in Finnish subjects. This variant has been shown to be associated with decreased IGF-1 concentrations, and IGF-1 has been shown to stimulate endothelial nitric oxide formation. ${ }^{18}$ Such observations might explain the association of rs34911341 with hypertension. ${ }^{11}$

In single-locus analysis, no other studied SNP was associated with hypertension or blood pressure. In particular, in agreement with other studies, ${ }^{12,19-21}$ we observed no association with the rs696217 (Leu72Met) variant. However, this variant was marginally associated with hypertension in a sample of obese women ${ }^{22}$ and has been shown to interact with dietary fat intake to modulate waist circumference and triglyceride concentrations ${ }^{23}$ and to modify the effect of physical activity on changes in weight and waist circumference. ${ }^{24}$ Although two studies failed to show any effect of this variant on plasma ghrelin concentrations, ${ }^{25,26}$ it cannot be ruled out that its effects, if any, can only be observed under specific environmental conditions. This could also explain why Mager et al. ${ }^{10}$ have observed an association between rs27647, rs26802, rs696217, rs4684677 and blood pressure levels in a population of overweight Finnish subjects with impaired glucose tolerance.

Interestingly, although rs26802 was not associated per se with hypertension in our study, we observed through a haplotype analysis that this polymorphism could modulate the risk of hypertension according to the haplotypes by which it is carried. The most likely explanation is that the observed haplotypic association might be due to other GHRL SNPs in linkage disequilibrium. Such a phenomenon may be an explanation for the discrepancy between results showing that this polymorphism is not associated with ghrelin levels ${ }^{25}$ but is associated with high-density lipoprotein cholesterol concentrations. ${ }^{24}$ According to the HapMap database, the studied SNPs cover about $75 \%$ of the haplotypic variability of the coding regions and proximal promoter of the GHRL gene. Further investigations and further genotyping would be required to completely characterize the genetic variability of the promoter region. Another limitation of our study is related to the blood pressure measurement methodology. Office-based one-point measurements may limit the accuracy of the blood pressure phenotype and therefore the selection of hypertensives vs. normotensives.

Conversely, no association was observed between any of the studied SNPs (or haplotypes) and atherosclerotic disease. These results are in agreement with studies that have reported a similar lack of association with cardiovascular disease in patients with type II diabetes mellitus ${ }^{12}$ or with $\mathrm{CHD} .^{13,27}$

In conclusion, this study provides statistical support for association of the GHRL gene with hypertension. Once clearly identified, the hypertension-associated GHRL SNPs might become useful markers in assessing the genetic risk of hypertension.

\section{CONFLICT OF INTEREST}

The authors declare no conflict of interest.

\section{ACKNOWLEDGEMENTS}

We thank Nadine Spenrath and Doris Vollmar for their excellent technical assistance. The study was sponsored in part by the Wilhelm Doerenkamp Foundation (Cologne, Germany). Lipid Analytic Cologne (LIANCO) was supported by Bayer Vital GmbH (Leverkusen, Germany).

1 Kojima M, Kangawa K. Ghrelin: structure and function. Physiol Rev 2005; 85: 495-522.

2 van der Lely AJ, Tschop M, Heiman ML, Ghigo E. Biological, physiological, pathophysiological, and pharmacological aspects of ghrelin. Endocr Rev 2004; 25: 426-457.

3 Wiedmer P, Nogueiras R, Broglio F, D'Alessio D, Tschop MH. Ghrelin, obesity and diabetes. Nat Clin Pract Endocrinol Metab 2007; 3: 705-712.

4 Li WG, Gavrila D, Liu X, Wang L, Gunnlaugsson S, Stoll LL, McCormick ML, Sigmund $\mathrm{CD}$, Tang C, Weintraub NL. Ghrelin inhibits proinflammatory responses and nuclear factor-kappaB activation in human endothelial cells. Circulation 2004; 109: 2221-2226.

5 Sharma V, McNeill JH. The emerging roles of leptin and ghrelin in cardiovascular physiology and pathophysiology. Curr Vasc Pharmacol 2005; 3: 169-180.

6 Tesauro M, Schinzari F, Iantorno M, Rizza S, Melina D, Lauro D, Cardillo C. Ghrelin improves endothelial function in patients with metabolic syndrome. Circulation 2005. 112: 2986-2992.

7 Katugampola SD, Pallikaros Z, Davenport AP. 125I-His(9)]-ghrelin, a novel radioligand for localizing GHS orphan receptors in human and rat tissue: up-regulation of receptors with athersclerosis. Br J Pharmacol 2001; 134: 143-149.

8 Poykko SM, Kellokoski E, Horkko S, Kauma H, Kesaniemi YA, Ukkola O. Low plasma ghrelin is associated with insulin resistance, hypertension, and the prevalence of type 2 diabetes. Diabetes 2003; 52: 2546-2553.

9 Poykko SM, Kellokoski E, Ukkola O, Kauma H, Paivansalo M, Kesaniemi YA, Horkko S. Plasma ghrelin concentrations are positively associated with carotid artery atherosclerosis in males. J Intern Med 2006; 260: 43-52.

10 Mager U, Kolehmainen M, Lindstrom J, Eriksson JG, Valle TT, Hamalainen $H$, Ilanne-Parikka P, Keinanen-Kiukaanniemi S, Tuomilehto JO, Pulkkinen L, Uusitupa MI. Association between ghrelin gene variations and blood pressure in subjects with impaired glucose tolerance. Am J Hypertens 2006; 19: 920-926.

11 Poykko S, Ukkola O, Kauma H, Savolainen MJ, Kesaniemi YA. Ghrelin Arg51GIn mutation is a risk factor for Type 2 diabetes and hypertension in a random sample of middle-aged subjects. Diabetologia 2003; 46: 455-458.

12 Ukkola 0, Kesaniemi YA. Preproghrelin Leu72Met polymorphism in patients with type 2 diabetes mellitus. J Intern Med 2003; 254: 391-394.

13 Tang NP, Wang LS, Yang L, Gu HJ, Zhu HJ, Zhou B, Sun QM, Cong RH, Wang B. Preproghrelin Leu72Met polymorphism in Chinese subjects with coronary artery disease and controls. Clin Chim Acta 2008; 387: 42-47.

14 Gouni-Berthold I, Giannakidou E, Müller-Wieland D, Faust M, Kotzka J, Berthold HK, Krone W. Association between the PPARalpha L162V polymorphism, plasma lipoprotein levels, and atherosclerotic disease in patients with diabetes mellitus type 2 and in nondiabetic controls. Am Heart J 2004; 147: 1117-1124.

15 Gouni-Berthold I, Giannakidou E, Müller-Wieland D, Faust M, Kotzka J, Berthold HK, Krone W. Peroxisome proliferator-activated receptor-gamma2 Pro12Ala and endothelial nitric oxide synthase- $4 \mathrm{a} / \mathrm{b}$ gene polymorphisms are not associated with hypertension in diabetes mellitus type 2. J Hypertens 2005; 23: 301-308.

16 Tregouet DA, Garelle V. A new JAVA interface implementation of THESIAS: testing haplotype effects in association studies. Bioinformatics 2007; 23: 1038-1039.

17 Vouyouka AG, Kent KC. Arterial vascular disease in women. J Vasc Surg 2007; 46: 1295-1302.

18 Böger RH, Skamira C, Bode-Böger SM, Brabant G, von zur Mühlen A, Frölich JC. Nitric oxide may mediate the hemodynamic effects of recombinant growth hormone in patients with acquired growth hormone deficiency. A double-blind, placebo-controlled study. J Clin Invest 1996; 98: 2706-2713.

19 Kim SY, Jo DS, Hwang PH, Park JH, Park SK, Yi HK, Lee DY. Preproghrelin Leu72Met polymorphism is not associated with type 2 diabetes mellitus. Metabolism 2006; 55 : 366-370.

20 Mager U, Lindi V, Lindstrom J, Eriksson JG, Valle TT, Hamalainen H, Ilanne-Parikka P, Keinanen-Kiukaanniemi S, Tuomilehto J, Laakso M, Pulkkinen L, Uusitupa M. Association of the Leu72Met polymorphism of the ghrelin gene with the risk of Type 2 diabetes in subjects with impaired glucose tolerance in the Finnish Diabetes Prevention Study. Diabet Med 2006; 23: 685-689.

21 Bing C, Ambye L, Fenger M, Jorgensen T, Borch-Johnsen K, Madsbad S, Urhammer SA. Large-scale studies of the Leu72Met polymorphism of the ghrelin gene in relation to the metabolic syndrome and associated quantitative traits. Diabet Med 2005; 22: 1157-1160.

22 Ukkola O, Ravussin E, Jacobson P, Perusse L, Rankinen T, Tschop M, Heiman ML, Leon AS, Rao DC, Skinner JS, Wilmore JH, Sjostrom L, Bouchard C. Role of ghrelin polymorphisms in obesity based on three different studies. Obes Res 2002; 10: 782-791. 
23 Robitaille J, Perusse L, Bouchard C, Vohl MC. Genes, fat intake, and cardiovascular disease risk factors in the Quebec Family Study. Obesity (Silver Spring) 2007; 15: 2336-2347.

24 Kilpelainen TO, Lakka TA, Laaksonen DE, Mager U, Salopuro T, Kubaszek A, Todorova B, Laukkanen O, Lindstrom J, Eriksson JG, Hamalainen H, Aunola S, Ilanne-Parikka P, Keinanen-Kiukaanniemi S, Tuomilehto J, Laakso M, Uusitupa $M$. Interaction of single nucleotide polymorphisms in ADRB2, ADRB3, TNF, IL6, IGF1R, LIPC, LEPR, and GHRL with physical activity on the risk of type 2 diabetes mellitus and changes in characteristics of the metabolic syndrome: The Finnish Diabetes Prevention Study. Metabolism 2008; 57 $428-436$.
25 Mager U, Kolehmainen M, de Mello VD, Schwab U, Laaksonen DE, Rauramaa R, Gylling H, Atalay M, Pulkkinen L, Uusitupa M. Expression of ghrelin gene in peripheral blood mononuclear cells and plasma ghrelin concentrations in patients with metabolic syndrome. Eur J Endocrinol 2008; 158: 499-510.

26 Berthold HK, Giannakidou E, Krone W, Mantzoros CS, Gouni-Berthold I. The Leu72Met polymorphism of the ghrelin gene is associated with a decreased risk for type 2 diabetes. Clin Chim Acta 2009; 399: 112-116.

27 Baessler A, Kwitek AE, Fischer M, Koehler M, Reinhard W, Erdmann J, Riegger G, Doering A, Schunkert H, Hengstenberg C. Association of the Ghrelin receptor gene region with left ventricular hypertrophy in the general population: results of the MONICA/ KORA Augsburg Echocardiographic Substudy. Hypertension 2006; 47: 920-927.

Supplementary Information accompanies the paper on Hypertension Research website (http://www.nature.com/hr) 